\title{
Musculoskeletal pain and work absence - a 10 year follow-up study of Norwegian young adult twins
}

\author{
Ragnhild E. Ørstavik ${ }^{1}$, Gun Peggy Knudsen ${ }^{1}$, Kristian Tambs ${ }^{1}$, Line C. Gjerde ${ }^{1,2}$, Fartein Ask \\ Torvik $^{1}$, Christopher Nielsen ${ }^{1,2}$, Espen Røysamb ${ }^{1,2}$ and Ted Reichborn-Kjennerud ${ }^{1,3}$ \\ 1) Domain for Mental and Physical Health, Norwegian Institute of Public Health \\ 2) Department of Psychology, University of Oslo \\ 3) Institute of Clinical Medicine, University of Oslo \\ E-mail: RagnhildElise.Orstavik@fhi.no
}

\begin{abstract}
Background and aims: Sickness absence (SA) and disability pension (DP) are increasingly recognized as major public problems. Musculoskeletal disorders are among the most common diagnoses set by physicians granting SA and DP. Results from recent twin studies have established that SA and DP are influenced not only by environmental and social factors, but also moderately to substantially by genes. The aim of the current study was to examine to what degree musculoskeletal complaints in young adults predict SA and DP, including SA granted for other diagnoses. As the participants were twins, we were able to perform within pair analyses, to see if the associations between musculoskeletal pain and later DP or SA were confounded by unmeasured genetic and shared environmental factors.

Materials and methods: The Norwegian twin registry includes a questionnaire conducted in 1998. From this, we included three measures of recurrent pain (lower back, neck/shoulders and muscular) as well as symptoms of anxiety and depression (measured by the Symptom Checklist-5 (SCL-5)). The questionnaire has been linked to highly reliable official registries on SA and DP, as well as a range of sociodemographic variables, for a ten-year follow up period. We applied logistic (DP as dependent variable) and binomial (SA as dependent variable) regression analyses to explore the relationship between musculoskeletal pain and DP and SA. In the final models, we adjusted for sociodemographic factors and symptoms of anxiety and depression. Differences between twins in a pair were explored by applying fixed effect models. All analyses were conducted using STATA version 13.1 .

Results: The final sample of 7,626 twins included 3,055 complete pairs (488 monozygotic (MZ) male, 349 dizygotic (DZ) male, $747 \mathrm{MZ}$ female, 589 DZ female, and 882 opposite sex twin pairs) and 1,516 singletons. By the end of follow up, 181 subjects ( 44 men and 137 women) received DP, and $63.7 \%$ of the sample (47.4\% of males and $76.0 \%$ of females) had at least one period of SA extending 16 days. Pain at any site was significantly associated with DP in both sexes. Any increase in the number of pain sites reported was associated with about a $60 \%$ increased risk for receiving DP (OR 1.6, 95\% CI 1.4-1.9), and the strength of the association was only marginally reduced when adjusted for symptoms of mental disorders (1.4, 1.21.7). In the within pair analyses the effect was no longer significant, indicating possible confounding from genetic and shared environmental effects. As for all cause SA, musculoskeletal pain predicted SA independently of all measured confounders, and the results remained significant in the within pair analyses (Incidence Rate Ratio (IRR) 1.12, 95\% CI 1.03-1.23).

Conclusion: In young adults, musculoskeletal pain strongly predicted SA and DP for a 10 year follow-up period. Musculoskeletal pain was associated with higher levels of all cause SA, even within discordant MZ twin pairs. Our results indicate that interventions to prevent musculoskeletal pain in young adults can reduce levels of SA and DP.
\end{abstract}

This is an open access article distributed under the Creative Commons Attribution Licence, which permits unrestricted use, distribution, and reproduction in any medium, provided the original work is properly cited.

Long term sickness absence (SA) and disability pensioning (DP) are increasingly recognized as major public health problems, especially when young adults with many potential working years are involved. Much effort has been made to gain a deeper understanding of health related, work related and societal factors underlying SA and DP, including, recently, large cohort studies of twins from the Nordic countries (Gjerde et al., 2013; Harkonmaki et al., 2008; Narusyte et al., 2011). Results from these studies have established that SA and DP are influenced not only by environmental and social factors, but also moderately to substantially by genes (Gjerde et al., 2013; Harkonmaki et al., 2008; Narusyte et al., 2011). Furthermore, epidemiological studies seeking to gain a deeper understanding of causal relationships have increasingly utilized genetically informative data, both to establish to what degree different phenotypes are influenced by genetic and environmental factors, and to control for potential confounding effects of genes and family environment when disentangling true causal effects from underlying confounders (McGue et al., 2010). Monozygotic (MZ) twins are genetically identical, whereas dizygotic (DZ) twins share on average $50 \%$ of their segregating genes. 
All twin pairs - regardless of zygosity - are matched on shared environmental factors, defined as environmental factors that make twins in a pair more similar to each other. Such shared environment will include socioeconomic status of their parents, shared neighborhood and schools, as well as certain intrauterine conditions including e.g. the mother's diet under pregnancy. Thus, for intra pair effects - that is, differences in outcome (discordance) within pairs are associated with differences in exposure within pairs or vice versa. In MZ twins all effects of genes and shared environment are controlled for, while for DZ twins this applies to $100 \%$ of shared environment and $50 \%$ of genetic effects (McGue et al., 2010).

Musculoskeletal disorders are among the leading causes of work absence and disability world-wide (Bevan, 2015; OECD, 2009), although mental disorders have become a more prominent cause during the past decades - especially among younger adults (OECD, 2009). These two groups of disorders are, however, not necessarily distinct as there is a well-recognized correlation between musculoskeletal complaints and symptoms of anxiety and depression (Goesling et al., 2013; Vargas-Prada \& Coggon, 2015). While it is highly disputed whether musculoskeletal or other forms of chronic pain leads to mental problems or vice versa, both phenotypes are found to be influenced by partially overlapping genetic factors (Goesling et al., 2013; Reichborn-Kjennerud et al., 2002).

So far, few population based studies have focused on the long-term association between musculoskeletal pain and SA and DP (Landmark, 2013; Nielsen, 2013; Ropponen et al., 2013). This is partly due to the lack of inclusion of these phenotypes in health surveys, and the scarcity of reliable data on DP and especially SA. In order to prevent disability pensioning and thereby most often permanent exclusion from the work force, it is, however, of crucial importance to focus on work participation and absence which in most cases (and perhaps especially the preventable ones) foregoes disability pensioning by many years. It is also of utmost importance to study populations of young adults those who are early in their working years and thereby establishing absence patterns that may proceed for the rest of their working life.

In the Norwegian Twin Registry, we have linked data from questionnaires and interviews, covering a variety of mental and physical health problems, to highly reliable official registries on SA and DP, as well as an array of socioeconomic measures. The aim of the current study was to explore the relationship between musculoskeletal complaints and subsequent SA and DP in a large, population-based cohort of young adults. As the participants were twins, we were able to explore within-pair differences to further explore causal pathways. When available, diagnoses from the medical forms underlying the reimbursements were included, thus making it possible to perform subanalyses of the relationship between musculoskeletal pain and SA and work absence granted for both somatic and mental disorders. Thus, our specific research questions were:

- To what degree does musculoskeletal pain predict DP and SA, granted for any reason, in young adults over a 10 -year period?

- To what degree does a history of musculoskeletal pain predict later SA granted for musculoskeletal diagnoses?

- To what degree does a history of musculoskeletal pain predict later SA granted for mental diagnoses, when symptoms of anxiety and depression are adjusted for?

- For all research questions, we aimed to adjust for potential confounders, including genetic and environmental factors shared by the twins.

\section{METHODS}

\section{Sample}

The sample for the current study originated from the Norwegian Institute of Public Health Twin Panel (NIPHTP). Twins were identified through the national Medical Birth Registry, established January 1, 1967. Participants were twins born between 1967 and 1979 who took part in a large questionnaire study in 1998, including questions on musculoskeletal pain, somatic disorders and mental health. By using national identification numbers issued to all Norwegians at birth, these data were linked to The Historical-Event Database (Norwegian: FD-Trygd) at Statistics Norway, providing longitudinal data on SA and DP from 1998 to 2008. The NIPHTP has been used previously in several studies, and is described thoroughly elsewhere (Harris et al., 2002, 2006; Nilsen et al., 2012; Nilsen et al., 2012).

Of the 12,700 twins invited to the questionnaire, $8,045(63 \%)$ responded after one reminder. Data from 7,710 were subsequently linked, as 335 twins had withdrawn from the NIPHTP or declined to take part in the current study. Further 12 twins were excluded due to lack of data on zygosity. We also decided to exclude 72 twins who received their DP pension before year 2000, as the pension would already have been obtained or in process at the time they answered the questionnaire.

Zygosity was initially determined using questionnaire items previously shown to correctly classify more than $97 \%$ of the twin pairs (Magnus et al., 1983), followed by DNA analyses on a subgroup of the sample. The discrepancy between classification based on the questionnaire and DNA markers implied an expected misclassification rate of less than $2 \%$ for the whole sample.

\section{Measures}

\section{Exposures}

Data on musculoskeletal pain was measured by three questions in the 1998 questionnaire: Under the heading "have you, or have you ever had, any of the following 
diseases or health problems" there were three questions related to musculoskeletal pain: "Recurrent neckand shoulder pain", "lumbar pain" and "longstanding muscular pain". Participants were also asked to answer which year their pain started and, if by then symptom free, the year the symptoms disappeared.

\section{Outcomes}

Data on SA and DP were provided from The Historical-Event Database (Norwegian: FD-Trygd) at Statistics Norway.

DP was constructed as a dichotomous variable, where all subjects who received DP during the followup period (part or full DP) were coded as on DP.

SAs exceeding 16 days are covered by the mandatory Norwegian Insurance Scheme for a duration of up to 52 weeks. Thus, the minimum SA period recorded in this study was 16 days. For each day from January 1, 1998 to December 31, 2008, we had information on whether each participant was registered as employed and at SA. Diagnosis set by physicians when SAs were granted were also available, coded according to the International Classification of Primary Care (WONCA, 2003). Three SA variables were constructed: SA granted for musculoskeletal disorders (diagnoses in the L-chapter), SA granted for mental disorders (diagnoses in the P-chapter) and SA granted for any disorder (including SA with missing diagnoses).

In order to account for multiple SA spells and varying degree of employment, we first constructed a ratio of days on SA divided by days eligible for SA, and then transformed this into a count variable (0-100) for the negative binomial regression analyses.

\section{Possible confounders}

Symptoms of mental disordes were measured by the Symptom Checklist-5 (SCL-5). This is a shortened version of SCL-25 (Hesbacher et al., 1980). The SCL-5 consists of five items addressing how the subject has felt for the last two weeks and has been found to correlate 0.92 with the full-scale version (Tambs \& Moum, 1993). We applied the recommended cut-off of 2.0 to define clinical symptoms of anxiety and depression.

\section{Statistical analyses}

To estimate the longitudinal association between musculoskeletal pain and DP, we ran logistic regression models with DP as dependent variable. Potential confounders were explored in univariate analyses, and entered into the model if they were significantly associated with the outcome variable. In these initial models, twins were treated as individuals, and standard errors were adjusted for dependency within pairs applying the vce (cl) option in STATA. This option relaxes the usual requirement that the observations be independent: The observations are independent across groups (clusters) but not necessarily within groups (twin pairs). The fully adjusted model was then repeated checking for within-pair differences, using a fixed effects model. A fixed effects model takes into account that the subjects included are twins, and the analyses are restricted to within pair effects. As MZ twins share all genetic and family environment, and DZ twins share on average $50 \%$ of their segregating genes and all their family environment, the method can cancel out unmeasured confounders that are due to shared genetic and environmental influences by including a between-pairs (fixed) effect. The remaining within-effect provides an estimate of the effect of musculoskeletal pain on DP, although unmeasured individual specific effects will still not be adjusted for. Influence of familial factors (genetic and common environment) is indicated if associations found in the analyses in the individual analyses change considerably in the fixed effects models. To be able to fully adjust for genetic confounding, and to disentangle between genetic and environmental confounding, analyses must be further stratified by zygosity. For details of the method applied, see e.g. Fujiwara \& Kawachi (2009).

The same strategy was applied to SA as outcome variable, using negative binomial regression as the SA data were over-dispersed (that is, the variance was higher than expected from a binomial distribution). These analyses were also repeated for each of the three SA categories: SA granted for any disorder, SA granted for musculoskeletal disorders and SA granted for mental disorders. After running the data with individuals as units, we ran within-pairs fixed effect analyses.

All analyses were performed in STATA version 13.1 .

\section{RESULTS}

\section{Sample characteristics}

The final sample of 7,626 twins included 3,055 complete pairs (488 monozygotic (MZ) male, 349 dizygotic (DZ) male, $747 \mathrm{MZ}$ female, $589 \mathrm{DZ}$ female, and 882 opposite sex twin pairs) and 1,516 singletons. Fifty-eight percent were female, and mean age in the beginning of the study (1998) was 25.5 years (range 19-31), thus 35.5 years (range 29-41) at the end of follow-up. Demographic details are given in Table 1.

\section{Musculoskeletal pain}

One in four participants had suffered musculoskeletal pain stemming from at least one pain site (Table 1). Women reported significantly more often pain at any site than men $(31.6 \%$ versus $22.3 \%, p<0.0001)$, and gender differences were especially prominent for neck/ shoulder and muscle pain.

Subjects with pain were significantly older (mean age in 199826.1 versus 25.5 years, $p<0.001$ ), had lower educational level and higher SCL-5 scores than those not reporting musculoskeletal pain (Table 1).

\section{DP}

In the total sample, 181 subjects (44 men and 137 women) received DP between year 2000 and 2008 


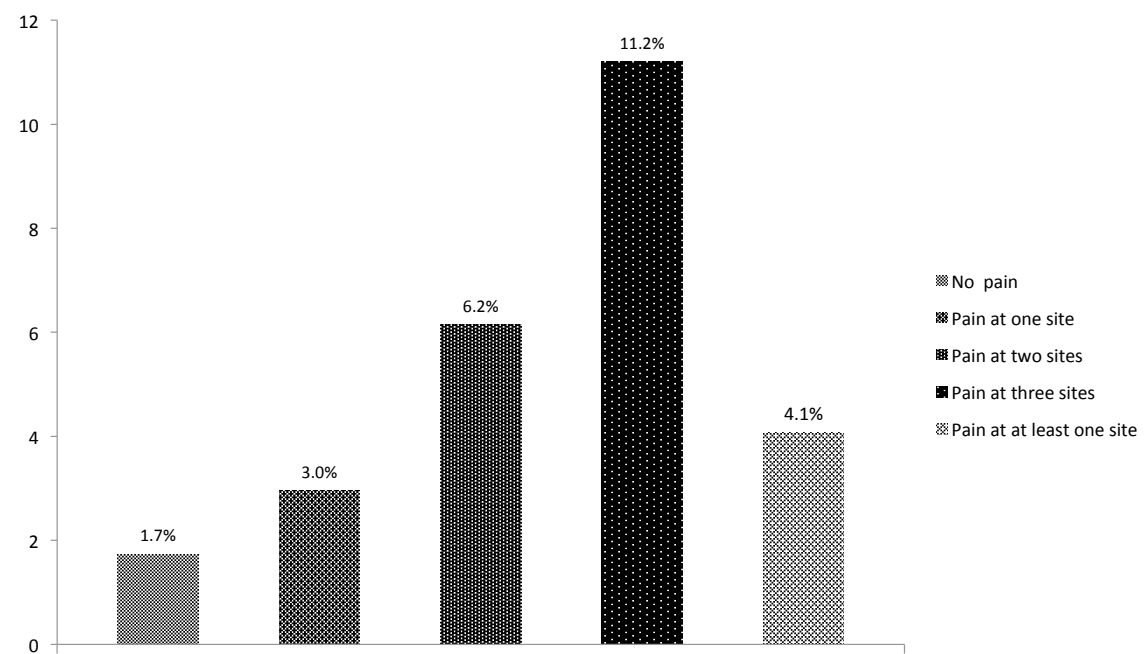

Figure 1. Number of pain sites.

Table 1. Sample characteristics.

\begin{tabular}{|c|c|c|c|c|c|c|}
\hline & \multicolumn{2}{|c|}{ All $(7,626)$} & \multicolumn{2}{|c|}{ No pain $(5,525)$} & \multicolumn{2}{|c|}{ Pain at any site $(2,101)$} \\
\hline Age in 1998 & \multicolumn{2}{|c|}{$25.5(3.7)$} & \multicolumn{2}{|c|}{$25.4(3.7)$} & \multicolumn{2}{|c|}{$25.9(3.6)$} \\
\hline Females & 4,408 & $57.8 \%$ & 3,023 & $54.7 \%$ & 1,385 & $65.9 \%$ \\
\hline \multicolumn{7}{|l|}{ Educational attainment (2008) } \\
\hline College degree or higher & 3,736 & $49.0 \%$ & 478 & $8.7 \%$ & 412 & $19.7 \%$ \\
\hline \multicolumn{7}{|l|}{ Musculoskeletal pain } \\
\hline Shoulder or neck pain & 1,013 & $13.3 \%$ & - & & & \\
\hline Low back pain & 1,453 & $19.1 \%$ & - & & & \\
\hline Muscular pain & 308 & $4.1 \%$ & - & & & \\
\hline Pain at any site & 2,101 & $27.6 \%$ & - & & & \\
\hline Pain at more than one site & 566 & $7.4 \%$ & - & & & \\
\hline \multicolumn{7}{|c|}{ Symptoms of anxiety and depression (SCL-5) } \\
\hline Clinical level (SCL-5 > 2.0) & 890 & $11.8 \%$ & 478 & $8.7 \%$ & 412 & $19.7 \%$ \\
\hline Disability pension & 181 & $2.4 \%$ & 96 & $1.7 \%$ & 85 & $4.1 \%$ \\
\hline \multicolumn{7}{|l|}{ Sick leave (any diagnosis) } \\
\hline No ( $0 \%$ of workdays) & 2,646 & $35.0 \%$ & 2,144 & $39.1 \%$ & 502 & $24.1 \%$ \\
\hline Some ( $0-2.9 \%$ of workdays $)$ & 1,639 & $21.7 \%$ & 1,258 & $23.0 \%$ & 381 & $18.3 \%$ \\
\hline Medium (2.9-10.2\% of workdays) & 1,641 & $21.7 \%$ & 1,137 & $20.8 \%$ & 504 & $24.2 \%$ \\
\hline High ( $>10.2 \%$ of workdays) & 1,637 & $21.6 \%$ & 939 & $17.2 \%$ & 698 & $33.5 \%$ \\
\hline \multicolumn{7}{|c|}{ Sick leave granted for musculoskeletal disorders } \\
\hline No $(0 \%$ of workdays $)$ & 5,248 & $69.4 \%$ & 4,057 & $74.1 \%$ & 1,191 & $57.1 \%$ \\
\hline Some ( $0-1.8 \%$ of workdays) & 772 & $10.2 \%$ & 520 & $9.5 \%$ & 252 & $12.1 \%$ \\
\hline Medium (1.8-6.2\% of workdays) & 771 & $10.2 \%$ & 514 & $9.4 \%$ & 257 & $12.3 \%$ \\
\hline High ( $>6.2 \%$ of workdays $)$ & 772 & $10.2 \%$ & 387 & $7.1 \%$ & 385 & $18.5 \%$ \\
\hline \multicolumn{7}{|l|}{ Sick leave granted for mental disorders } \\
\hline No $(0 \%$ of workdays $)$ & 6,201 & $82.0 \%$ & 4,617 & $84.3 \%$ & 1,548 & $76.0 \%$ \\
\hline Some ( $0-1.8 \%$ of workdays) & 454 & $6.0 \%$ & 306 & $5.6 \%$ & 148 & $7.1 \%$ \\
\hline Medium (1.8-7.1\% of workdays) & 454 & $6.0 \%$ & 290 & $5.3 \%$ & 164 & $7.9 \%$ \\
\hline $\operatorname{High}(>7.1 \%$ of workdays $)$ & 454 & $6.0 \%$ & 265 & $4.8 \%$ & 189 & $9.1 \%$ \\
\hline
\end{tabular}

(Table 1). Pain at any site was significantly associated with DP in both sexes. The relative frequency of DP increased with increasing number of pain sites (Figure 1). DP was also associated with lower educational level, female sex and an SCL-5 score above clinical levels (all p-values below 0.001). These variables were thus treated as confounders.

We first restricted the number of adjustments made in the multivariate analyses to sex, age and educational level, and then included SCL-5. In the first model, any increase in the number of pain sites reported was associated with about a $60 \%$ increased risk for receiving DP (OR 1.6, 95\% CI 1.4-1.9). The strength of the association was marginally reduced when adjusted for SCL-5 (1.4, 1.2-1.7). In the within pair analysis, which was restricted to pairs discordant for DP ( $n=144$ pairs) 
Table 2. Binomial regression analyses - the association between musculoskeletal pain and sick leave (incidence rate ratios (IRRs) and 95\% CI).

\begin{tabular}{lccccc}
\hline & Model 1 & Model 2 & Model 3 & Model 4 & Model 5 \\
& $\mathrm{N}=7,563$ & $\mathrm{~N}=7,506$ & $\mathrm{~N}=4,778$ & $\mathrm{~N}=2,930$ & $\mathrm{~N}=1,848$ \\
\hline All sick leave & 1.56 & 1.43 & 1.20 & 1.24 & 1.12 \\
& $(1.45-1.67)$ & $(1.34-1.53)$ & $(1.13-1.27)$ & $(1.15-1.34)$ & $(1.03-1.23)$ \\
Sick leave granted for musculoskeletal disorders & 1.96 & 1.93 & 1.27 & 1.35 & 1.12 \\
& $(1.76-2.18)$ & $(1.73-2.15)$ & $(1.16-1.38)$ & $(1.21-1.52)$ & $(0.97-1.28)$ \\
Sick leave granted for mental disorders & 1.61 & 1.12 & 1.00 & 1.02 & 0.97 \\
& $(1.35-1.93)$ & $(0.97-1.29)$ & $(0.88-1.13)$ & $(0.87-1.19)$ & $(0.80-1.18)$ \\
\hline
\end{tabular}

Model 1: adjusted for age, sex and educational attainment

Model 2: adjusted for age, sex, educational attainment and symptoms of mental disorders

Model 3: intrapair analyses (all twins), adjusted for sex, educational attainment and symptoms of mental disorders

Model 4: intrapair analyses, DZ twins, adjusted for sex, educational attainment and symptoms of mental disorders

Model 5: intrapair analyses, MZ twins, adjusted for educational attainment and symptoms of mental disorders

the association was no longer significant (OR 1.16, 95\% CI 0.79-1.69). Due to the low numbers, subanalyses on zygosity groups were not performed.

\section{$S \boldsymbol{A}$}

During the nine year follow-up period, $65.0 \%$ of the sample (48.4\% of males and $77.0 \%$ of females) had at least one period of SA extending 16 days. Musculoskeletal disorders were the most common diagnosis set for SA, and accounted for $34.7 \%$ of all SA days. Mental disorders accounted for $22.5 \%$, while no diagnosis was registered in $11.8 \%$ of lost days.

In total, $30.6 \%$ of the sample had at least one period of SA diagnosed with a musculoskeletal disorder, while $18.0 \%$ had at least one period diagnosed with a mental disorder (Table 1).

The results of the multivariate analyses are displayed in Table 2. In the total sample, musculoskeletal pain was independently associated with later all cause SA and for SA granted for both musculoskeletal and mental disorders. For example, one unit increase in pain sites was associated with an increase in SA granted for mental disorders with a factor of $1.61(95 \%$ CI 1.36-1.90). Adjusting for symptoms of anxiety and depression left the estimate for SA granted for musculoskeletal pain almost unchanged. When adjusting for symptoms of mental disorders in the analyses of SA due to mental disorders, the effect of pain was no longer statistically significant (IRR 1.12 , 95\% CI 0.95 1.30). These results were similar in the intrapair analyses, although the effect sizes decreased and - in MZ twins - the association between musculoskeletal pain and later SA due to musculoskeletal disorders was no longer significant.

\section{DISCUSSION}

In this longitudinal study of a large sample of Norwegian twins, we found that three brief questions on musculoskeletal pain was associated with a higher risk of DP and increased levels of SA in the following ten years. This applied also when our results were adjusted for co-occuring symptoms of anxiety and depression. In the analyses restricted to twin pair differences it appeared that some of the association might be confounded by genes and environmental factors shared by the twins.

There is a general lack of general epidemiological studies on the impact of musculoskeletal pain on future SA. To the best of our knowledge, this is the first study on the association where young adult twins are used as the study sample, and thus the possibility of adjusting of unmeasured confounders. Most studies have focused on musculoskeletal pain as a predictor for future disability (Landmark, 2013; Ropponen et al., 2011; Ropponen et al., 2013), and even here current knowledge is sparse and our ability to compare our results with those obtained previously limited. In a cross-sectional study, a subsample of 3,421 subjects from the Norwegian HUNT-study (HUNT 3) was invited to report pain every three months during a 12 month period (Landmark, 2013). As in our study, data was combined with registry based information on income, education and DP awards. Compared to subjects without chronic pain, the probability of being a receiver of DP was four times higher for those with chronic pain. Similar results was obtained when using data from survey conducted by Statistics Norway (Nielsen, 2013), in a sample of 1,694 subjects aged 2664. Measurement of pain in the HUNT and SSB studies were not restricted to musculoskeletal sites, but included migraine and other forms of chronic headache. Taking into account the differences in age between our sample and those reported above, and the different analytic strategies, it is interesting that we obtained comparable results and that the relationship between pain and work attendance can be extended to SA as well as DP.

In a Swedish twin sample, data on musculoskeletal pain (low back, neck and shoulder) for more than 11,000 twins born before 1958 were collected in 1975 and again in 1981 (Ropponen et al., 2013). In this survey, pain assessment was restricted to those who reported that their symptoms impaired their ability to 
work. Such pain was a strong risk factor for future DP granted for musculoskeletal disorders (low back pain or osteoarthritis). DP granted for other diagnoses was not included. Most associations held true also in the co twin control analyses, so the authors concluded that work impairing musculoskeletal pain was associated with DP independently of a number of possible confounders including genetics and family environment. It is interesting that pain assessed in our study, without reference to impairment and in young adults only, showed similar results to those obtained in the Swedish sample.

In a more recent, general epidemiologic study from Sweden, the authors did not include measures of pain but instead combined data from several registries to explore to what degree the interplay between back pain and mental disorders predicted DP (Dorner et al., 2016). Back pain was not measured directly, but defined as present if the subject were on SA due to back pain or had been to specialist care for the same diagnoses. A similar approach (although including antidepressant use) was applied to identify subjects with common mental disorders. In this study, adjusted HR for DP was approximately 4.0 for subjects reporting low back pain, and the authors found a synergistic effect between the two disorders. We chose a rather different approach, with self-reported musculoskeletal pain and SCL as predictor variables, and our cohort included young adults only. Again, it is interesting that studies using so different strategies still find similar results with regard to the association between musculoskeletal pain complaints or disorders and DP.

SA and DP are closely related, so our results for SA can also be regarded in light of previous findings on DP. We were not surprised to find that musculoskeletal pain predicted SA due to all causes or to musculoskeletal disorders in particular. The fact that this association was not significant in our within pair analyses of MZ twins (for musculoskeletal disorders, see Table 2, Model 5) can have several explanations. It is possible that this association is mediated by genetic factors, and that there is no "true" association. A perhaps more plausible explanation would be that we did not measure musculoskeletal pain more than once, and that a huge part of MZ cotwins developed such symptoms during the follow-up period. This would mean that having a genetically identical cotwin with musculoskeletal pain is indeed a risk factor of similar magnitude to suffering from such pain yourself. A third explanation might be that we did not have sufficient statistical power to demonstrate a true effect, given that it was rather moderate (IRR $1.12,95 \% \mathrm{CI}$ 0.97-1.28).

We were rather surprised to find that musculoskeletal pain did not seem to predict SA due to mental disorders - when SCL scores were adjusted for (IRR 1.12, 95\% CI 0.95-1.30). As mentioned above, musculoskeletal pain and symptoms of anxiety and depression are highly correlated, and given that we only measured these symptoms once, we would expect that many of those suffering from musculoskeletal pain would later develop symptoms of mental disorders. There are, however, many possible explanations for this finding. The most likely might be that somatic disorders are regarded with less stigma than mental disorders, so that patients with musculoskeletal pain and anxiety and depression who needs to be absent from work are more likely to get a musculoskeletal diagnosis than one for depression or anxiety.

\section{Strengths and limitations}

The strengths of our study include population based data, prospective design and the use of high quality registries to obtain reliable outcome data. Our results must, however, be interpreted in light of several limitations: First, our measurements of musculoskeletal pain and our most important confounder, symptoms of mental disorders, were measured only once and rather briefly. More reliable data on musculoskeletal pain, especially regarding time of onset, duration and strength, would have strengthened our data. The use of sharts to describe locations might also have improved our data - as well as, and perhaps more importantly, multiple time points of measurement. As described above, we do not know whether subjects who reported no musculoskeletal complaints in 1998 might have developed such health problems at a time closer to follow-up. Second, our sample size was limited and the prevalence of DP low. Due to the low prevalence of DP, we were not able to perform subanalyses on DP due to various disorders, and the results of our within pair analyses must be interpreted with caution. Reduced power is a well-recognized limitation when applying all kinds of within pair analyses, as samples are reduced to complete pairs, and further, in case of digitomized variables, to discordant pairs - and then further split on zygosity. Another limitation in discordant twin pair analyses is that discordant pairs might differ more often than others on other, unmeasured factors than the outcome (Madsen \& Osler, 2009). Third, our data did not include subjects who were granted DP without first having been employed, or those who for other reasons were excluded from the labor marked. Fourth, our results might not be applicable to other countries, especially those outside Scandinavia, as SA and DP depend on social and political factors as well as health.

\section{CONCLUSION}

In young adults, brief questions on musculoskeletal pain strongly predicted SA and DP for a 10 year follow-up period, and the associations seem to be partly independent of a wide range of measured and unmeasured confounders. Our results indicate that interventions to prevent musculoskeletal pain in young adults can reduce levels of SA and DP. 


\section{REFERENCES}

Bevan, S. (2015). Economic impact of musculoskeletal disorders (MSDs) on work in Europe. Best Pract Res Clin Rheumatol, 29 (3), 356-373. doi: 10.1016/j.berh.2015.08.002.

Dorner, T. E., Alexanderson, K., Svedberg, P., Tinghog, P., Ropponen, A., \& Mittendorfer-Rutz, E. (2016). Synergistic effect between back pain and common mental disorders and the risk of future disability pension: a nationwide study from Sweden. Psychol Med, 46 (2), 425-436. doi: 10.1017/s003329171500197x.

Fujiwara, T., \& Kawachi, I. (2009). Is education causally related to better health? A twin fixed-effect study in the USA. Int J Epidemiol, 38 (5), 1310-1322. doi: 10.1093/ije/dyp226.

Gjerde, L. C., Knudsen, G. P., Czajkowski, N., Gillespie, N., Aggen, S. H., Roysamb, E., et al. (2013). Genetic and environmental contributions to long-term sick leave and disability pension: A population-based study of young adult Norwegian twins. Twin Res Hum Genet, 16 (4), 759-766. doi: 10.1017/Thg.2013.36.

Goesling, J., Clauw, D. J., \& Hassett, A. L. (2013). Pain and depression: an integrative review of neurobiological and psychological factors. Curr Psychiatry Rep, 15 (12), 421. doi: 10.1007/s11920-013-0421-0.

Harkonmaki, K., Silventoinen, K., Levalahti, E., Pitkaniemi, J., Huunan-Seppala, A., Klaukka, T., et al. (2008). The genetic liability to disability retirement: a 30-year follow-up study of 24,000 Finnish twins. PLoS One, 3 (10), e3402.

Harris, J. R., Magnus, P., \& Tambs, K. (2002). The Norwegian Institute of Public Health Twin Panel: a description of the sample and program of research. Twin Res, 5 (5), 415-423.

Harris, J. R., Magnus, P., \& Tambs, K. (2006). The Norwegian Institute of Public Health twin program of research: an update. Twin Res Hum Genet, 9 (6), 858-864.

Hesbacher, P. T., Rickels, K., Morris, R. J., Newman, H., \& Rosenfeld, H. (1980). Psychiatric illness in family practice. J Clin Psychiatry, 41 (1), 6-10.

Landmark, T. Romundstad, P., Dale, O., Borchgrevink, P.C., Vatten, L., Kaasa, S. (2013). Chronic pain: One year prevalence and associated characteristics (the HUNT pain study). Scand J Pain, 4, 182-187.

Madsen, M., \& Osler, M. (2009). Commentary: Strengths and limitations of the discordant twin-pair design in social epidemiology. Where do we go from here? Int J Epidemiol, 38 (5), 1322-1323. doi: 10.1093/ije/dyp264.

Magnus, P., Berg, K., \& Nance, W. E. (1983). Predicting zygosity in Norwegian twin pairs born 1915-1960. Clin Genet, 24 (2), 103-112.

McGue, M., Osler, M., \& Christensen, K. (2010). Causal inference and observational research: the utility of twins. Perspect Psychol Sci, 5 (5), 546-556.

Narusyte, J., Ropponen, A., Silventoinen, K., Alexanderson, K., Kaprio, J., Samuelsson, A., \& Svedberg, P. (2011). Genetic liability to disability pension in women and men: a prospective population-based twin study. PLoS One, 6 (8), e23143.

Nielsen, C. S. (2013). Chronic pain is strongly associated with work disability. Scand J Pain, 4, 180-181.

Nilsen, T. S., Brandt, I., Magnus, P., \& Harris, J. R. (2012). The Norwegian Twin Registry. Twin Res Hum Genet, 15 (6), 775-780.

Nilsen, T. S., Knudsen, G. P., Gervin, K., Brandt, I., Roysamb, E., Tambs, K., et al. (2012). The Norwegian Twin Registry from a public health perspective: A research update. Twin Res Hum Genet, 1-11.

OECD (2009). Sickness, Disability and Work - Keeping on Track in the Economic Downturn. Retrieved from http://www.oecd.org/els/emp/42699911.pdf.

Reichborn-Kjennerud, T., Stoltenberg, C., Tambs, K., Roysamb, E., Kringlen, E., Torgersen, S., \& Harris, J. R. (2002). Back-neck pain and symptoms of anxiety and depression: a population-based twin study. Psychol Med, 32 (6), 1009-1020.

Ropponen, A., Silventoinen, K., Svedberg, P., Alexanderson, K., Koskenvuo, K., Huunan-Seppala, A., et al. (2011). Health-related risk factors for disability pensions due to musculoskeletal diagnoses: a 30-year Finnish twin cohort study. Scand J Public Health, 39 (8), 839-848.

Ropponen, A., Svedberg, P., Kalso, E., Koskenvuo, M., Silventoinen, K., \& Kaprio, J. (2013). A prospective twin cohort study of disability pensions due to musculoskeletal diagnoses in relation to stability and change in pain. Pain, 154 (10), 1966-1972. doi: 10.1016/j.pain.2013.05.029.

Tambs, K., \& Moum, T. (1993). How well can a few questionnaire items indicate anxiety and depression? Acta Psychiatr Scand, 87 (5), 364-367.

Vargas-Prada, S., \& Coggon, D. (2015). Psychological and psychosocial determinants of musculoskeletal pain and associated disability. Best Pract Res Clin Rheumatol, 29 (3), 374-390. doi: 10.1016/j.berh.2015.03.003.

WONCA, I. C. C. (2003). International Classification of Primary Care, Second edition (ICPC-2). Retrieved 02.01.2016, 2016. 